\title{
CONTROL AND STABILITY ANALYZING OF THE TIME-DELAY SYSTEMS WITH TIME-VARYING DELAYS
}

\author{
Daniel GONTKOVIČ, Róbert FÓNOD \\ Department of Cybernetics and Artificial Intelligence, Faculty of Electrical Engineering and Informatics, Technical University of \\ Košice, Letná 9, 04200 Košice, Slovak Republic, tel.: +421 55625 2749, e-mail: daniel.gontkovic@tuke.sk, \\ robert.fonod@student.tuke.sk
}

\begin{abstract}
Systems with delays are a specific group of dynamic systems. The fact that some delays can be short and some can be long makes system analysis and control design much more complex. In this paper we present a proposal to solve the problem of stability and control design of continuous time systems with time-varying delays. Based on the Lyapunov-Krasovskii functional the stability condition is derived using the linear matrix inequality (LMI) techniques, and convex optimization principle to find LMI variables which simultaneously satisfies the limitations given by the theory of Lyapunov-Krasovskii functionals. Obtained solution is the feasible convex LMI problem for the static controller design. Finally the design method is demonstrated using a system model example.
\end{abstract}

Keywords: Lyapunov-Krasovskii functional, LMI, Lyapunov function, Time-delay systems, Time-varying delays, Schur complement

\section{INTRODUCTION}

Systems with delays frequently appear in engineering, where time delays are the property of a physical system. Whenever material, information or energy is physically transmitted from one place to another, there is a delay associated with the transmission. The value of the delay is determined by the distance and the transmission speed, where some delays are short, some are very long. Since delays might lead to a system destabilization, additive conditions, based on the assumption that in the system there is information on delays states, are taking into account in design task formulation. Such systems are called systems with delayed state variables. However, the presence of long delays makes system analysis and control design substantially much more complex.

The study of functional differential equations started long before 1900, but the mathematical formulation of the problems were developed in the 20th century. Thus, the notion of a functional differential equation was introduced by Myshkis [13] in 1949. A further progress have been made to study systems with delays in the past 50 years ([14], [2] and the references therein); main ideas concerning the work in this area can be found in e.g. Krasovskii [8] (time-domain approach, extension of the Lyapunov second method to functional differential equations). Burton [1] (refinements of the Lyapunov-Krasovskii theory and periodic solutions) During the last decades, we have witnessed significant development in the control of time-delay systems. Some recent comprehensive introductions to the problem are in Greeki [5], Marshall et al. [12], Kolmanovskii and Myshkis [7] and Hale and Lunel [6].

In this paper the design task of the stabilizing controller for the closed-loop system is transmuted into LMI framework and solved. There are two ways to use the second method of Lyapunov for time-delay systems. The first is based on the theory of Lyapunov-Krasovskii functionals and the other is based on the theory of LyapunovRazumikhin functions. The paper extends the control design and stability analyzing methods based on theory of Lyapunov-Krasovskii functionals with limited speed of change of time delays on the interval $\beta=\langle 0,1)$.

\section{SYSTEM MODEL}

Equations of linear dynamic systems with time delay are as follows

$\dot{\mathbf{q}}(t)=\mathbf{A q}(t)+\mathbf{A}_{d} \mathbf{q}(t-\tau(t))+\mathbf{B u}(t)$

$\mathbf{y}(t)=\mathbf{C q}(t)$

$0 \leq \tau(t) \leq \tau_{m}<\infty, 0<\dot{\tau}(t) \leq \beta<1, \tau_{m}, \beta \in \mathbb{R}$

with the initial condition

$\mathbf{q}(\vartheta)=\varphi(\vartheta), \vee \vartheta \in\left\langle-\tau_{m}, 0\right\rangle$

where $\tau(t)>0$ is the time-varying delay of state, $\mathbf{q}(t) \in \mathbb{R}^{n}$ is a vector of the state, $\mathbf{u}(t) \in \mathbb{R}^{r}$ and $\mathbf{y}(t) \in \mathbb{R}^{m}$ are vectors of the input and output variables, $\mathbf{A} \in \mathbb{R}^{n \times n}$ is the nominal system matrix, $\mathbf{A}_{d} \in \mathbb{R}^{n \times n}, \mathbf{B} \in \mathbb{R}^{n \times r}, \mathbf{C} \in \mathbb{R}^{m \times n}$.

Problem of the interest is to design stable close-loop system with the linear memoryless state feedback controller of the form

$\mathbf{u}(t)=-\mathbf{K} \mathbf{q}(t)$,

where $t \geq 0$ and $\mathbf{K} \in \mathbb{R}^{r \times n}$ is the controller gain matrix.

\section{BASIC PRELIMINARIES}

Working with the linear matrix inequalities the concept of symmetric positive definite matrix or positive semidefinite matrix is principally used. The necessary and sufficient conditions for positive definiteness of a symmetric matrix $\mathbf{P}$ are:

- there is a regular matrix $\mathbf{Q}$, such as that $\mathbf{P}=\mathbf{Q}^{T} \mathbf{Q}$

- all eigenvalues of the matrix $\mathbf{P}$ are positive,

- all main subdeterminants of the matrix $\mathbf{P}$ are positive $\left|\mathbf{p}_{11}\right|>0,\left|\begin{array}{ll}\mathbf{p}_{11} & \mathbf{p}_{12} \\ \mathbf{p}_{21} & \mathbf{p}_{22}\end{array}\right|>0, \ldots, \operatorname{det}(\mathbf{P})>0$. 
Working with the norm, in the next $\|\cdot\|$ denotes the standard Euclidean norm, and $\|\cdot\|_{\infty}$ the $H_{\infty}$ norm.

Proposition 3.1. (Schur Complement)

If $\boldsymbol{Q}=\boldsymbol{Q}^{T}>0, \boldsymbol{R}=\boldsymbol{R}^{T}, \boldsymbol{S}$ are real matrices of appropriate dimensions, then the next inequalities are equivalent

$$
\begin{gathered}
{\left[\begin{array}{cc}
\boldsymbol{Q} & \boldsymbol{S} \\
\boldsymbol{S}^{T} & \boldsymbol{R}
\end{array}\right]>0 \Leftrightarrow\left[\begin{array}{cc}
\boldsymbol{Q}-\boldsymbol{S R}^{-1} \boldsymbol{S}^{T} & \boldsymbol{0} \\
\boldsymbol{0} & \boldsymbol{R}
\end{array}\right]>0} \\
\boldsymbol{Q}-\boldsymbol{S \boldsymbol { R } ^ { - 1 }} \boldsymbol{S}^{T}>0, \quad \boldsymbol{R}>0 .
\end{gathered}
$$

Proof. (e.g. see $[9,10]$ )

Let the linear matrix inequalities takes form

$$
\left[\begin{array}{cc}
\mathbf{Q} & \mathbf{S} \\
\mathbf{S}^{T} & \mathbf{R}
\end{array}\right]>0
$$

than using Gauss elimination it yields

$$
\begin{gathered}
{\left[\begin{array}{cc}
\mathbf{I} & -\mathbf{S R}^{-1} \\
\mathbf{0} & \mathbf{I}
\end{array}\right]\left[\begin{array}{cc}
\mathbf{Q} & \mathbf{S} \\
\mathbf{S}^{T} & \mathbf{R}
\end{array}\right]\left[\begin{array}{cc}
\mathbf{I} & \mathbf{0} \\
-\mathbf{R}^{-1} \mathbf{S}^{T} & \mathbf{I}
\end{array}\right]=} \\
=\left[\begin{array}{cc}
\mathbf{Q}-\mathbf{S R}^{-1} \mathbf{S}^{T} & \mathbf{0} \\
\mathbf{0} & \mathbf{R}
\end{array}\right]
\end{gathered}
$$

and it is evident that this transformation does not change positivity (6), and so (7) implies (5).

Definition 3.1. (Lyapunov function $[11,15]$ )

For some $\tau(t)>0$ the equilibrium 0 of system (1), (2) is:

- uniformly stable if there exists a positive definite continuous functional $v\left(\boldsymbol{q}_{t}(\boldsymbol{\theta})\right)$ whose derivative $\dot{v}\left(\boldsymbol{q}_{t}(\theta)\right)$ is negative semi-definite functional;

- uniformly asymptotically stable if there exists a positive definite upper-bounded continuous functional $v\left(\boldsymbol{q}_{t}(\boldsymbol{\theta})\right)$, whose derivative $\dot{v}\left(\boldsymbol{q}_{t}(\boldsymbol{\theta})\right)$ is negative definite functional;

- uniformly exponentially stable if there exist a positive definite continuous functional $v\left(\boldsymbol{q}_{t}(\theta)\right)$ and positive real constants $\alpha, \beta, \gamma, \delta$ such that $\alpha\left\|\boldsymbol{q}_{t}(\theta)\right\| \leq$ $v\left(\boldsymbol{q}_{t}(\theta)\right) \leq \beta\left\|\boldsymbol{q}_{t}(\boldsymbol{\theta})\right\|, \quad \dot{v}\left(\boldsymbol{q}_{t}(\theta)\right) \leq-\gamma\left\|\boldsymbol{q}_{t}(\boldsymbol{\theta})\right\|$, and $\left.\mid v \boldsymbol{q}_{t}\left(\theta_{1}\right)\right)-v\left(\boldsymbol{q}_{t}\left(\theta_{2}\right)\right) \mid \leq \delta\left\|\boldsymbol{q}_{t}\left(\theta_{1}\right)-\boldsymbol{q}_{t}\left(\theta_{2}\right)\right\|$.

Proposition 3.2. (Lyapunov-Krasovskii Ineqality [3, 4])

The linear time delay system of form (1), (2) is stable with quadratic performance $\left\|\boldsymbol{C}(s \boldsymbol{I}-\boldsymbol{A})^{-1} \boldsymbol{B}\right\|_{\infty}=\lambda$ for $\dot{\tau}(t) \leq \beta$ if exists a symmetric positive definite matrix $\boldsymbol{P}$ and $\boldsymbol{Q}$ and a scalar variable $\gamma>0$ such that

$$
\begin{aligned}
& {\left[\begin{array}{cccc}
\boldsymbol{A}^{T} \boldsymbol{P}+\boldsymbol{P A}+\boldsymbol{Q} & \boldsymbol{P A}_{d} & \boldsymbol{P B} & \boldsymbol{C}^{T} \\
* & -(1-\beta) \boldsymbol{Q} & \boldsymbol{0} & \boldsymbol{0} \\
* & * & -\gamma \boldsymbol{I}_{r} & \boldsymbol{0} \\
* & * & * & -\boldsymbol{I}_{m}
\end{array}\right]<0} \\
& \boldsymbol{P}=\boldsymbol{P}^{T}>0, \quad \boldsymbol{Q}=\boldsymbol{Q}^{T}>0, \quad \gamma>0
\end{aligned}
$$

Hereafter, * denotes the symmetric item in a symmetric matrix.

The problem is reduced to solving LMI i.e. to find matrices $\mathbf{P}$ and $\mathbf{Q}, \mathbf{P} \in \mathbb{R}^{n \times n}, \mathbf{Q} \in \mathbb{R}^{n \times n}$, and a scalar variable $\gamma>0$, which simultaneously satisfies the boundary (8).
Proof. Defining Lyapunov-Krasovskii functional candidate as follows

$$
\begin{gathered}
v(\mathbf{q}(t), t)=\mathbf{q}^{T}(t) \mathbf{P q}(t)+\int_{t-\tau(t)}^{t} \mathbf{q}^{T}(r) \mathbf{Q q}(r) \mathrm{d} r+ \\
+\int_{0}^{t}\left(\mathbf{y}^{T}(r) \mathbf{y}(r)-\gamma \mathbf{u}^{T}(r) \mathbf{u}(r)\right) \mathrm{d} r>0 \\
\mathbf{P}>0, \quad \mathbf{Q}>0, \quad \gamma>0
\end{gathered}
$$

Using Laplace transform, let

$$
\tilde{\mathbf{y}}(s)=\mathbf{G}(s) \tilde{\mathbf{u}}(s),\|\mathbf{G}(s)\|_{\infty}=\left\|\mathbf{C}(s \mathbf{I}-\mathbf{A})^{-1} \mathbf{B}\right\|_{\infty}=\lambda
$$

when $\tilde{\mathbf{y}}(s), \tilde{\mathbf{u}}(s)$ stand for Laplace transform of $m$ dimensional output vector and $r$ dimensional input vector signal, respectively and $\mathbf{G}(s)$ is the transfer function matrix.

Then Parcevall's theorem implies

$$
\frac{\left[\int_{0}^{\infty} \mathbf{y}^{T}(t) \mathbf{y}(t) \mathrm{d} t\right]^{\frac{1}{2}}}{\left[\int_{0}^{\infty} \mathbf{u}^{T}(t) \mathbf{u}(t) \mathrm{d} t\right]^{\frac{1}{2}}}=\frac{\|\mathbf{y}(t)\|}{\|\mathbf{u}(t)\|} \leq \lambda .
$$

Since $H_{\infty}$ norm can be interpreted as a maximum gain in any direction and at any frequency then there exists a scalar $\gamma \lambda^{2}>0$ such that Lyapunov-Krasovskkii functional candidate be positive definite.

The result of the functional derivative of $v(\mathbf{q}(t), t)$ is

$$
\begin{gathered}
\dot{v}(\mathbf{q}(t), t)= \\
=\dot{\mathbf{q}}^{T}(t) \mathbf{P q}(t)+\mathbf{q}^{T}(t) \mathbf{P} \dot{\mathbf{q}}(t)+\mathbf{q}^{T}(t) \mathbf{Q q}(t)- \\
-\mathbf{q}^{T}(t-\tau(t)) \mathbf{Q q}(t-\tau(t))(1-\dot{\tau}(t))+ \\
\quad+\mathbf{y}^{T}(t) \mathbf{y}(t)-\gamma \mathbf{u}^{T}(t) \mathbf{u}(t)<0
\end{gathered}
$$

Inserting (1) into (12) gives

$$
\begin{gathered}
\left(\mathbf{A q}(t)+\mathbf{A}_{d} \mathbf{q}(t-\tau(t))+\mathbf{B} \mathbf{u}(t)\right)^{T} \mathbf{P} \mathbf{q}(t)+ \\
+\mathbf{q}^{T}(t) \mathbf{P}\left(\mathbf{A} \mathbf{q}(t)+\mathbf{A}_{d} \mathbf{q}(t-\tau(t))+\mathbf{B} \mathbf{q}(t)\right)+ \\
+\mathbf{q}^{T}(t) \mathbf{Q q}(t)-(1-\dot{\tau}(t)) \mathbf{q}^{T}(t-\tau(t)) \mathbf{Q q}(t-\tau(t))+ \\
+\mathbf{q}^{T}(t) \mathbf{C}^{T} \mathbf{C} \mathbf{q}(t)-\gamma \mathbf{u}^{T}(t) \mathbf{u}(t)<0
\end{gathered}
$$

where

$$
\mathbf{y}^{T}(t) \mathbf{y}(t)=\mathbf{q}^{T}(t) \mathbf{C}^{T} \mathbf{C q}(t) .
$$

Creating a composite vector $\mathbf{q}^{\circ}(t)$

$\mathbf{q}^{\circ T}(t)=\left[\begin{array}{lll}\mathbf{q}^{T}(t) & \mathbf{q}^{T}(t-\tau(t)) & \mathbf{u}^{T}(t)\end{array}\right]$

the inequality $(13)$ can be rewritten in a form

$\mathbf{q}^{\circ T}(t)\left[\begin{array}{ccc}\Delta_{11}^{\bullet} & \mathbf{P A}_{d} & \mathbf{P B} \\ * & -(1-\beta) \mathbf{Q} & \mathbf{0} \\ * & * & -\gamma \mathbf{I}_{r}\end{array}\right] \mathbf{q}^{\circ}(t)$

where $\Delta_{11}^{\bullet}=\mathbf{A}^{T} \mathbf{P}+\mathbf{P A}+\mathbf{Q}+\mathbf{C}^{T} \mathbf{C}$.

Using Schur complement property it is possible to write

$$
\left[\begin{array}{cccc}
\mathbf{A}^{T} \mathbf{P}+\mathbf{P A}+\mathbf{Q} & \mathbf{P A}_{d} & \mathbf{P B} & \mathbf{C}^{T} \\
* & -(1-\beta) \mathbf{Q} & \mathbf{0} & \mathbf{0} \\
* & * & -\gamma \mathbf{I}_{r} & \mathbf{0} \\
* & * & * & -\mathbf{I}_{m}
\end{array}\right]<0 .
$$

It is obvious that (17) implies (8). 


\section{PARAMETER DESIGN}

Theorem 4.1. For system (17), (2) the necessary and sufficient condition for the stable nominal control (4) with quadratic performance $\| \boldsymbol{C}(\text { sI }-\boldsymbol{A})^{-1} \boldsymbol{B} \|_{\infty}=\lambda$ is that there exist positive definite symmetric matrices $\boldsymbol{X}, \boldsymbol{Z}$, matrix $\boldsymbol{Y}$ and a scalar variable $\gamma>0$, such that the following LMIs are satisfied

$$
\left.\begin{array}{ccccc}
\Delta_{11} & \boldsymbol{X} & \boldsymbol{A}_{d} \boldsymbol{Z} & \boldsymbol{B} & \boldsymbol{X} \boldsymbol{C}^{T} \\
* & -\boldsymbol{Z} & \boldsymbol{0} & \boldsymbol{0} & \boldsymbol{0} \\
* & * & -(1-\beta) \boldsymbol{Z} & \boldsymbol{0} & \boldsymbol{0} \\
* & * & * & -\gamma \boldsymbol{I}_{r} & \boldsymbol{0} \\
* & * & * & * & -\boldsymbol{I}_{m}
\end{array}\right]<0
$$

where $\Delta_{11}=\boldsymbol{X} \boldsymbol{A}^{T}+\boldsymbol{A} \boldsymbol{X}-\boldsymbol{B} \boldsymbol{Y}-\boldsymbol{Y} \boldsymbol{B}^{T}$.

The control law gain matrix is then given as

$\boldsymbol{K}=\boldsymbol{Y} \boldsymbol{X}^{-1}$.

Proof. The linear state feedback control law, defined in (4) gives rise to the closed-loop system as follows

$\dot{\mathbf{q}}(t)=(\mathbf{A}-\mathbf{B K}) \mathbf{q}(t)+\mathbf{A}_{d} \mathbf{q}(t-\tau(t))$.

Subsituting in (8) gives for $\mathbf{P}=\mathbf{P}^{T}>0$ and $\mathbf{Q}=\mathbf{Q}^{T}>0$

$\left[\begin{array}{cccc}\Delta_{11} & \mathbf{P A}_{d} & \mathbf{P B} & \mathbf{C}^{T} \\ * & -(1-\beta) \mathbf{Q} & \mathbf{0} & \mathbf{0} \\ * & * & -\gamma \mathbf{I}_{r} & \mathbf{0} \\ * & * & * & -\mathbf{I}_{m}\end{array}\right]<0$

where $\Delta_{11}^{\bullet}=(\mathbf{A}-\mathbf{B K})^{T} \mathbf{P}+\mathbf{P}(\mathbf{A}-\mathbf{B K})+\mathbf{Q}$.

Defining the congruence transform matrix

$$
\mathbf{T}_{1}=\mathbf{T}_{1}^{T}=\operatorname{diag}\left[\begin{array}{llll}
\mathbf{P}^{-1} & \mathbf{I} & \mathbf{I} & \mathbf{I}
\end{array}\right]
$$

and premultiplying 21 from the left and right side by $\mathbf{T}_{1}$ gives

$\left[\begin{array}{cccc}\Delta_{11}^{\circ} & \mathbf{A}_{d} & \mathbf{B} & \mathbf{P}^{-1} \mathbf{C}^{T} \\ *-(1-\beta) \mathbf{Q} & \mathbf{0} & \mathbf{0} \\ * & * & -\gamma \mathbf{I}_{r} & \mathbf{0} \\ * & * & * & -\mathbf{I}_{m}\end{array}\right]<0$

where $\Delta_{11}^{\circ}=\mathbf{P}^{-1}(\mathbf{A}-\mathbf{B K})^{T}+(\mathbf{A}-\mathbf{B K}) \mathbf{P}^{-1}+\mathbf{P}^{-1} \mathbf{Q} \mathbf{P}^{-1}$.

Substituting $\mathbf{P}^{-1}=\mathbf{X}$ and $\mathbf{Y}=\mathbf{K} \mathbf{P}^{-1}$ then 23 can be rewriten such that

$\left[\begin{array}{cccc}\Delta_{11}^{\diamond} & \mathbf{A}_{d} & \mathbf{B} & \mathbf{X C}^{T} \\ * & -(1-\beta) \mathbf{Q} & \mathbf{0} & \mathbf{0} \\ * & * & -\gamma \mathbf{I}_{r} & \mathbf{0} \\ * & * & * & -\mathbf{I}_{m}\end{array}\right]<0$

where $\Delta_{11}^{\diamond}=\mathbf{X A}^{T}+\mathbf{A X}-\mathbf{B Y}-\mathbf{Y B}^{T}+\mathbf{X Q X}$.

Using the Schur complement (24) can be modified as

$$
\left[\begin{array}{ccccc}
\Delta_{11} & \mathbf{X} & \mathbf{A}_{d} & \mathbf{B} & \mathbf{X C}^{T} \\
* & -\mathbf{Q}^{-1} & \mathbf{0} & \mathbf{0} & \mathbf{0} \\
* & * & -(1-\beta) \mathbf{Q} & \mathbf{0} & \mathbf{0} \\
* & * & * & -\gamma \mathbf{I}_{r} & \mathbf{0} \\
* & * & * & * & -\mathbf{I}_{m}
\end{array}\right]<0
$$

where $\Delta_{11}=\mathbf{X} \mathbf{A}^{T}+\mathbf{A X}-\mathbf{B Y}-\mathbf{Y B}^{T}$.

Defining the next congruence transform matrix

$$
\mathbf{T}_{2}=\mathbf{T}_{2}{ }^{T}=\operatorname{diag}\left[\begin{array}{lllll}
\mathbf{I} & \mathbf{I} & \mathbf{Q}^{-1} & \mathbf{I}_{r} & \mathbf{I}_{m}
\end{array}\right]
$$

and then premultiplying (25) from the right and left side by 26) modify it in the form

$$
\left[\begin{array}{ccccc}
\Delta_{11} & \mathbf{X} & \mathbf{A}_{d} \mathbf{Q}^{-1} & \mathbf{B} & \mathbf{X C}^{T} \\
* & -\mathbf{Q}^{-1} & \mathbf{0} & \mathbf{0} & \mathbf{0} \\
* & * & \Delta_{33} & \mathbf{0} & \mathbf{0} \\
* & * & * & -\gamma \mathbf{I}_{r} & \mathbf{0} \\
* & * & * & * & -\mathbf{I}_{m}
\end{array}\right]<0
$$

where $\Delta_{33}=-(1-\beta) \mathbf{Q}^{-1}$. The substitution $\mathbf{Q}^{-1}=\mathbf{Z}$ leads to inequality

$$
\left[\begin{array}{ccccc}
\Delta_{11} & \mathbf{X} & \mathbf{A}_{d} \mathbf{Z} & \mathbf{B} & \mathbf{X C}^{T} \\
* & -\mathbf{Z} & \mathbf{0} & \mathbf{0} & \mathbf{0} \\
* & * & -(1-\beta) \mathbf{Z} & \mathbf{0} & \mathbf{0} \\
* & * & * & -\gamma \mathbf{I}_{r} & \mathbf{0} \\
* & * & * & * & -\mathbf{I}_{m}
\end{array}\right]<0 .
$$

It is obvious that (28) implies (18).

\section{ILLUSTRATIVE EXAMPLE}

To demonstrate the algorithm properties it was assumed that system is given by (1), (2), where

$\mathbf{A}=\left[\begin{array}{rrr}-2.6 & 0.0 & 0.8 \\ -1.2 & 0.2 & 0.0 \\ 0.0 & 0.5 & -3.0\end{array}\right], \mathbf{C}=\left[\begin{array}{ll}1 & 1 \\ 2 & 1 \\ 1 & 0\end{array}\right]$,

$\mathbf{A}_{d}=\left[\begin{array}{rrr}0.00 & 0.02 & 0.00 \\ 0.00 & 0.00 & -1.00 \\ -0.02 & 0.00 & 0.00\end{array}\right], \mathbf{B}=\left[\begin{array}{ll}4 & 0 \\ 7 & 1 \\ 1 & 0\end{array}\right]$.

Solving (18) with respect to the LMI matrix variables $\mathbf{X}>0, \mathbf{Z}>0, \mathbf{Y}$ and a scalar variable $\gamma>0$ using SeDuMi package for Matlab, given task was feasible with

$$
\begin{aligned}
& \mathbf{X}=\left[\begin{array}{rrr}
1.3892 & -0.5363 & -0.0136 \\
-0.5363 & 0.9210 & -0.2723 \\
-0.0136 & -0.2723 & 1.1760
\end{array}\right], \\
& \mathbf{Z}=\left[\begin{array}{rrr}
3.9383 & -0.0842 & 0.0013 \\
-0.0842 & 3.8726 & -0.0286 \\
0.0013 & -0.0286 & 3.0627
\end{array}\right], \\
& \mathbf{Y}=\left[\begin{array}{rrc}
-0.1671 & 2.0496 & -0.2288 \\
-0.1352 & 0.4042 & 0.5026
\end{array}\right], \quad \gamma=4.6235,
\end{aligned}
$$

where $\mathbf{P}^{-1}=\mathbf{X}$ and $\mathbf{Q}^{-1}=\mathbf{Z}$. The controller gain matrix $\mathbf{K}_{1}$ can be obtained from the relation 19 for $\beta=0.1$, where

$\mathbf{K}_{1}=\left[\begin{array}{lll}1.0341 & 2.9774 & 0.5068 \\ 0.1885 & 0.7253 & 0.5975\end{array}\right]$,

$\rho\left(\mathbf{A}_{u}\right)=\rho\left(\mathbf{A}-\mathbf{B K}_{1}\right)=\{-11.0867,-2.0799,-2.7745\}$

It is evident that the eigenvalues spectrum $\rho\left(\mathbf{A}_{u}\right)$ of the closed control loop is stable.

Setting $\beta=0.5$ the gain matrix $\mathbf{K}_{2}$ takes the form

$\mathbf{K}_{2}=\left[\begin{array}{lll}1.1799 & 3.3692 & 0.5428 \\ 0.1997 & 0.8179 & 0.6076\end{array}\right]$, 
$\rho\left(\mathbf{A}_{u}\right)=\rho\left(\mathbf{A}-\mathbf{B K}_{2}\right)=\{-12.4348,-2.0624,-2.7705\}$

and the spectrum of the eigenvalues $\rho\left(\mathbf{A}_{u}\right)$ of the closed control loop is stable, too.

Solving with respect to the controller gain matrix $\mathbf{K}_{3}$ and $\beta=0.99$ results

$$
\mathbf{K}_{3}=\left[\begin{array}{ccc}
2.3169 & 5.3545 & 1.2816 \\
0.3283 & 1.1451 & -0.8732
\end{array}\right] \text {. }
$$

Even for this case is the eigenvalues spectrum $\rho\left(\mathbf{A}_{u}\right)$ of the closed control loop is stable, since

$\operatorname{eig}\left(\mathbf{A}_{u}\right)=\{-18.8092,-1.4764,-4.2613\}$

\subsection{Simulation}

Time-delay linear dynamic system (1), (2) is raised up to the steady state starting with the nonzero initial conditions $[-1,0.5,3]^{T}$.

In Figure 1 to 3 is shown the time response of the closed-loop system with respect to the stability region conditioned by the delay time changes interval $0<\dot{\tau}(t) \leq 1$. Simulations were made with the prescribed values $\beta=0.1$, $\beta=0.5, \beta=0.99$ from above defined interval. Note, the closed-loop system is $t \in\langle-\tau(t), 0)$ unstable, because one's eigenvalue of $\mathbf{A}_{d}$ positive. Closed-loop system reaches the steady state values of output variables approximately

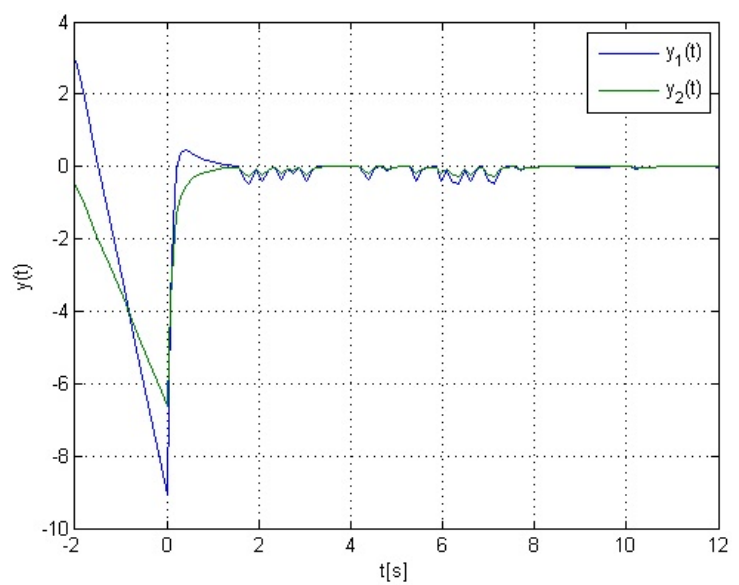

in time instants less then $11 \mathrm{~s}$. It can be easily seen that the criterion proposed in this paper to design the memoryless feedback controller gives acceptable solutions. Moreover, the limited range of the delay time changes interval $0<\dot{\tau}(t) \leq 1$ is given by the necessary negativeness of the Lyapunov-Krasovskii functional derivative.

\section{CONCLUDING REMARKS}

Time delays can appear as a part of the dynamics in many technological processes. Presence of delays in the system is making the analysis and control design more complex because it has a negative impact on the stability of system. Tendency to instability generally grows with respect to the size of the time delay. Therefore, the proposal of ensuring stability and control design systems with delays is a very interesting topic in the scientific community.

The method uses the standard LMI numerical optimization procedures to manipulate the system feedback gain matrix as the direct design variable. Since it is necessary to propose an asymptotically stable closed-loop system, to solve the control design problem in the sense of delayindependent criteria, we typically use such modifications of Lyapunov-Krasovskii functional as shown above. An example, illustrating the design method certifies the presented principle is applicable.

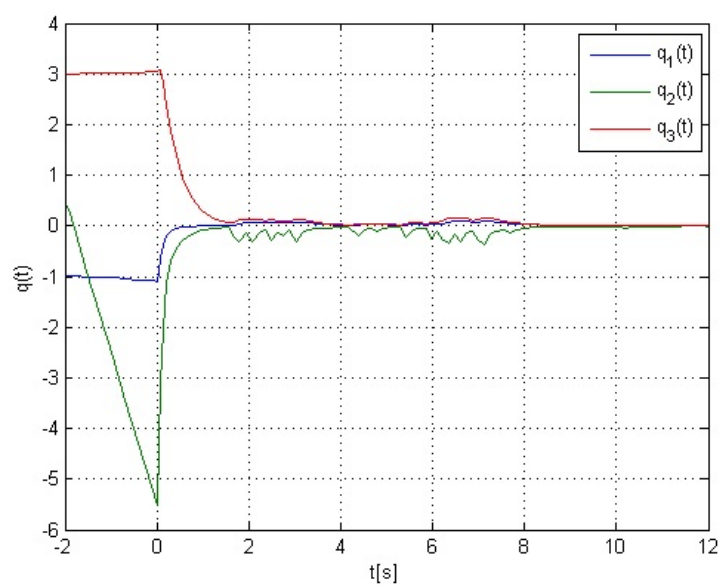

Fig. 1 Response of the closed-loop system for $\beta=0.1$

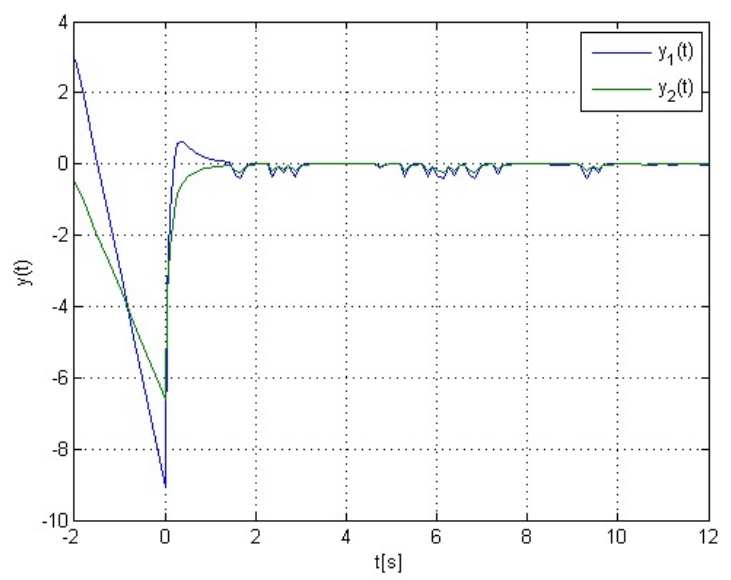

Fig. 2 Response of the closed-loop system for $\beta=0.5$ 

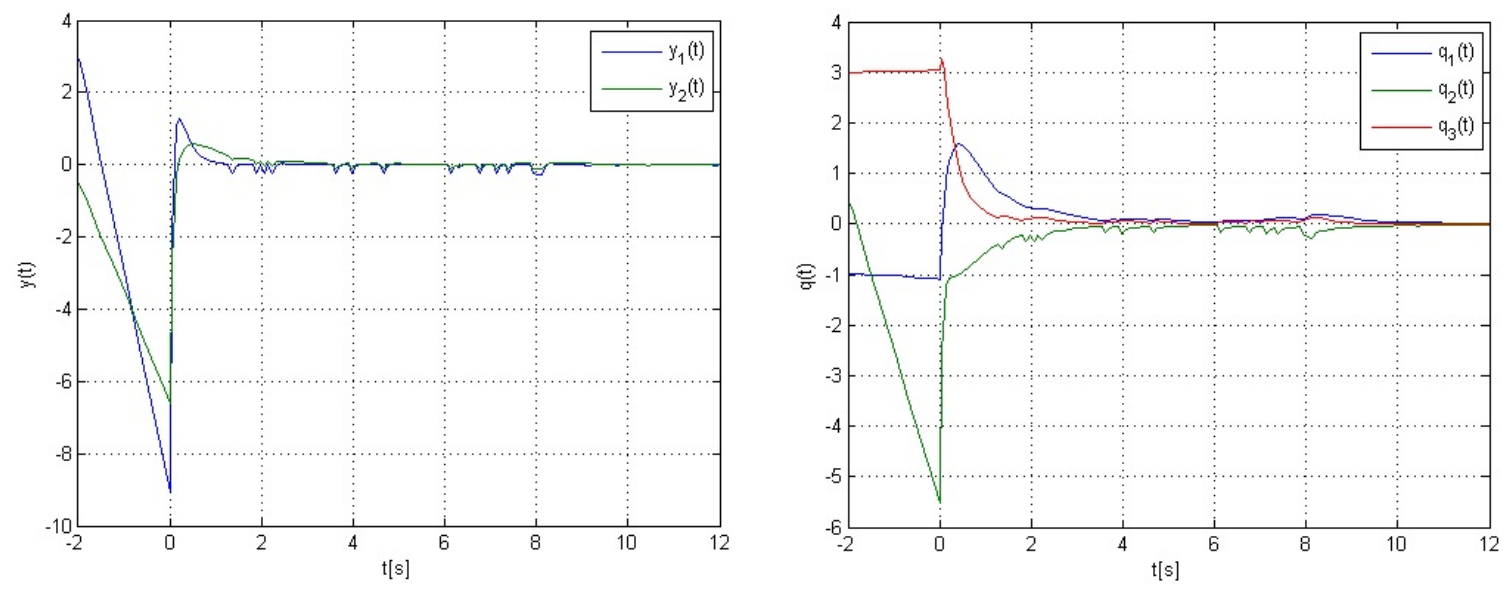

Fig. 3 Response of the closed-loop system for $\beta=0.99$

\section{ACKNOWLEDGEMENT}

The work presented in this paper was supported by VEGA, Grant Agency of Ministry of Education and Academy of Science of Slovak Republic under Grant No. $1 / 0256 / 11$. This support is very gratefully acknowledged.

\section{REFERENCES}

[1] BURTON, T. A.: Stability and periodic solutions of ordinary and functional differential equations, Orlando: Academic Press, 1985.

[2] DIBLÍK, J. et al.: Control of Oscillating Systems with a Single Delay. Hindawi Publishing Corporation Advances in Difference Equations, 2010, Art. ID 108218, 15 pp., DOI:10.1155/2010/108218.

[3] FILASOVÁ, A. - KROKAVEC, D.: Asymptotically stable control design for time-delay systems, in Proceedings of the 17th International Conference Process Control '09, June 9-12, 2009, Štrbské Pleso, Slovak Republic, pp. 358-362.

[4] FILASOVÁ, A. - KROKAVEC, D.: Global asymptotically stable control design for time-delay systems. AT\&P Journal Plus, No. 2, 2009, pp. 89-92.

[5] GÓRECKI, H. et al.: Analysis and Synthesis of Time Delay Systems, Warszawa: John Wiley \& Sons (PWN), 1989.

[6] HALE, J. K. - VERDUYN LUNEL, S. M.: Introduction to Functional Differential Equations, New York: Springer-Verlag, 1991.

[7] KOLMANOVSKII, V. - MYSHKIS, A.: Applied Theory of Functional Differential Equations, Dordrecht: Kluwer, 1992.

[8] KRASOVSKII, N. N.: Stability of motion: Application of Lyapunov's Second Method to Differential Systems and Equations with Delay, Standford: Stanford University Press, 1963.

[9] KROKAVEC, D. - FILASOVÁ, A.: Optimal Stochastic Systems, Košice: ELFA, s.r.o., 2002. (in Slovak)
[10] KROKAVEC, D. - FILASOVÁ, A.: Discrete-time Systems, Elfa, Košice, 2008. (in Slovak)

[11] KROKAVEC, D. - FILASOVÁ, A.: Exponential stability of networked control systems with networkinduced random delays. Archives of Control Sciences, Vol. 20, No. 2, 2010, pp. 165-186.

[12] MARSHALL, J. E. et al.: Time-delay systems: Stability and performance criteria with applications. New York: Ellis Horwood, 1992.

[13] MYSHKIS, A. D.: General theory of differential equations with delay. Uspechi Matematicheskich Nauk, (Engl. Transl.), AMS, 1951.

[14] NICULESCU, S.I. - VERRIEST, E.I. - DUGARD, L. - DION, J.M. : Stability and robust stability of timedelay systems: A guided tour, In Stability and Control of Time-Delay Systems, DUGARD L. and VERRIEST E.I. (eds.), London: Springer-Verlag, 1998, pp. 1-71.

[15] SUN, Z. - GE, S. S.: Switched Linear Systems, Control and Design, London: Springer-Verlag, 2005.

Received March 4, 2011, accepted July 8, 2011

\section{BIOGRAPHIES}

Daniel Gontkovič was born in 1984 in Poprad, Slovakia. He graduated in automation in 2009 from the Faculty of Electrical Engineering and Informatics, Technical University of Košice, Slovakia. Since 2009 he is a PhD. student with the Department of Cybernetics and Artificial Intelligence, FEI TU Košice. His scientific research is focusing on time-delay systems and system control.

Róbert Fónod was born in 1987 in Veľký Krtíš, Slovakia. He received BSc. degree in Cybernetics in 2009 from the Faculty of Electrical Engineering and Informatics, Technical University of Košice. Since 2009 he is a MSc. student in the same study branch with the Department of Cybernetics and Artificial Intelligence, FEI TU Košice. His interest is in dynamic system fault diagnostics. 